\title{
Xanthatin inhibits corneal neovascularization by inhibiting the VEGFR2-mediated STAT3/PI3K/Akt signaling pathway
}

\author{
MEI SHEN ${ }^{1,2^{*}}$, XUE-ZHI ZHOU ${ }^{3 *}$, LEI YE ${ }^{1}$, QING YUAN $^{1}$, CE SHI $^{4}$, PEI-WEN ZHU ${ }^{1}$, \\ NAN JIANG ${ }^{1,2}$, MING-YANG MA ${ }^{1}$, QI-CHEN YANG ${ }^{2}$ and YI SHAO ${ }^{1}$ \\ ${ }^{1}$ Department of Ophthalmology, The First Affiliated Hospital of Nanchang University, \\ Jiangxi Province Clinical Ophthalmology Institute, Nanchang, Jiangxi 330006; \\ ${ }^{2}$ Eye Institute of Xiamen University, Xiamen, Fujian 361102; \\ ${ }^{3}$ Department of Ophthalmology, Xiangya Hospital, Central South University, Changsha, Hunan 410008; \\ ${ }^{4}$ School of Ophthalmology and Optometry, Wenzhou Medical University, Wenzhou, Zhejiang 325035, P.R. China
}

Received August 9, 2017; Accepted April 26, 2018

DOI: 10.3892/ijmm.2018.3646

\begin{abstract}
Alkali burn is one of the main causes of corneal injury. The inflammation and neovascularization caused by alkali burns aggravate corneal damage, resulting in loss of vision. The aim of the present study was to evaluate the efficacy of xanthatin in the treatment of alkali burn-induced inflammation and neovascularization. A CCK-8 assay was used to detect the effects of different concentrations of xanthatin on the proliferation of human umbilical vein endothelial cells (HUVECs). The effects of xanthatin on the migration of HUVECs and the ability of lumen formation were examined using a scratch test and lumen formation assay, respectively. A total of 60 Sprague-Dawley rats were randomly divided into two groups to establish a corneal alkali burn model, and were treated with PBS and xanthatin eye drops four times a day. A slit lamp microscope recorded changes of the cornea at $0,4,7,10$ and 14 days, and the inflammatory indices of the cornea and the neovascular area were evaluated. The expression levels of vascular endothelial growth factor (VEGF) and pigment epithelium-derived factor (PEDF) in the cornea under different treatment conditions were detected using immunofluorescence and western blot analysis. In order to investigate the mechanism of xanthatin on the inhibition of inflammation and neovascularization, HUVECs were treated with xanthatin and PBS following VEGF treatment. The subcellular localization
\end{abstract}

Correspondence to: Professor Yi Shao, Department of Ophthalmology, The First Affiliated Hospital of Nanchang University, Jiangxi Province Clinical Ophthalmology Institute, 17 Yongwaizheng Street, Donghu, Nanchang, Jiangxi 330006, P.R. China

E-mail: freebee99@163.com

*Contributed equally

Key words: xanthatin, cornea, neovascularization, inflammation, alkali-burn injury of signal transducer and activator of transcription 3 (STAT3) was detected using immunofluorescence. The expression levels of VEGF receptor 2 (VEGFR2), STAT3, phosphoinositide 3-kinase (PI3K) and Akt were detected using western blot analysis. The results revealed that xanthatin inhibited the proliferation of HUVECs in a concentration-dependent manner. The migration ability and lumen-forming ability of the HUVECs were also inhibited by xanthatin. Slit lamp microscopy showed that the inflammatory index and the area of neovascularization in the xanthatin-treated group were significantly reduced, compared with those in the PBS treatment group. The xanthatin treatment group exhibited a lower protein expression level of VEGF and increased protein expression level of PEDF, compared with the PBS treatment group. In the VEGF-treated HUVECs, xanthatin significantly decreased the expression levels of p-VEGFR2, phosphorylated (p-)STAT3, p-PI3K and p-Akt. In conclusion, the present study confirmed that xanthatin inhibited corneal neovascularization and inflammation in the alkali burn model, elucidating the underlying mechanisms involved in its protective effects. Therefore, xanthatin may be a novel drug for the treatment of corneal alkali burn.

\section{Introduction}

As an important feature of the anterior corneal refractive component, normal corneal function is indispensable for the formation of visual function and protection of the eye. Under hypoxia, inflammation, trauma and other harmful conditions, inflammatory cells secrete a variety of angiogenic chemokines, including basic fibroblast growth factor, vascular endothelial growth factor (VEGF) and prostaglandin, which lead to endothelial cell proliferation, differentiation, migration and chemotaxis. This promotes angiogenesis invasion from the corneal limb vascular network, which affects corneal transparency and eventually leads to blindness (1). According to the World Health Organization statistics, corneal disease is currently the second leading cause of disease-related blindness, whereas keratitis and neovascularization are the major causes of corneal damage-induced blindness. Current clinical 
studies are mainly focused on the identification of novel anti-inflammatory and anti-angiogenic drugs (2).

There are 25 Xanthium species in the world (3). At present, xanthatin is the main medicinal component of Xanthium in China, which is used for the treatment of cold headaches, nasal mucus, itching, rhinorrhea and muscular constriction (4). Sesquiterpene lactone is a biologically active component of medicinal plants. The sesquiterpene lactone components of Xanthium sibiricum often contain $\alpha, \beta$ unsaturated carbonyl y-lactone-y fragments, which have beneficial biological activities, including antimicrobial, antioxidant, anti-allergy, anti-inflammatory, hypoglycemic and other pharmacological effects (2). Xanthatin is the main active constituent of sesquiterpene lactones in Xanthium sibiricum. It has antitumor (5), antifungal (6) and antiplasmodium (7) activities, and has attracted substantial attention in widespread studies.

Neovascularization is the process of vascular instability, proliferation and migration of vascular endothelial cells. At present, there are at least six different associated growth factors involved in the process of neovascularization, in which VEGF is the most important angiogenic factor. The effect of VEGF on vascular endothelial cells is predominantly regulated by two types of tyrosine kinase receptors, VEGF receptor (VEGFR)1 and VEGFR2 $(8,9)$. VEGFR2 is more important in regulating the mitosis and penetration of endothelial cells $(10,11)$. Xanthatin can effectively inhibit the VEGFR2 signaling pathway, thereby inhibiting angiogenesis and reducing inflammation (12). In addition, xanthatin can exert anti-inflammatory effects by inhibiting the synthesis of proinflammatory substances nitric oxide, tumor necrosis factor- $\alpha$ and prostaglandin E2, and the activity of 5-lipoxygenase (13).

Corneal alkali burn is a model of a severe ocular surface disease, which can lead to corneal ulcers, severe keratitis, corneal neovascularization and corneal scarring. Therefore, it is widely used to investigate the mechanism and treatment of corneal inflammation and angiogenesis. In the present study, the application of xanthatin eye drops was used for the treatment of corneal alkali burn, and the effects of xanthatin on inflammation and neovascularization were evaluated. The experimental results showed that xanthatin inhibited corneal neovascularization, reduced inflammation and assisted in repair of the corneal epithelium. Therefore, xanthatin is expected to be an effective drug for the treatment of keratitis and neovascularization.

\section{Materials and methods}

Cell culture. Human umbilical vein endothelial cells (HUVECs) which were obtained from the Cell Line Bank of the Chinese Academy of Sciences (Shanghai, China) were cultured with endothelial cell basal medium 2 (ECM2) containing nonessential amino acids, vitamins, growth factor, $2 \%$ FCS (Thermo Fisher Scientific, Inc., Waltham, MA, USA) and $1 \%$ penicillin/streptomycin at $37^{\circ} \mathrm{C}$ in an atmosphere containing $5 \% \mathrm{CO}_{2}$.

CCK-8 assay for cell proliferation. The passage 3-6 adherent HUVECs were washed with PBS and digested into cell suspension with $0.25 \%$ trypsin (EDTA) cells, and seeded in 96-well plates $\left(5 \times 10^{3}\right.$ cells/well $)$ in eight groups. The cells were cultured under $5 \% \mathrm{CO}_{2}$ in $37^{\circ} \mathrm{C}$ for $24 \mathrm{~h}$, and $100-\mu \mathrm{l}$ volumes of different concentrations of xanthatin in ECM 2 were added. The final concentrations of xanthatin were $0.1,0.5,1,2,5,10$ and $20 \mu \mathrm{M}$. The cells were washed once with PBS following culture for $72 \mathrm{~h}$, and were then incubated with serum-free ECM2 with $10 \mu \mathrm{l} \mathrm{CCK}-8$ solution for $1 \mathrm{~h}$. The OD $450 \mathrm{~nm}$ absorbance was measured using a microplate spectrophotometer.

Scratch assay for cell migration. When the cells (1x10 $/$ well) were grown to almost confluence, the culture medium in 24-well plates was replaced with serum-free medium for $24 \mathrm{~h}$ and cells were divided into two groups: A control group and a $10 \mu \mathrm{M}$ xanthatin treatment group. A sterile scratch was made with a $200-\mu 1$ tip to establish a cell wound model. The width of the wound was measured under phase contrast microscopy. Images were captured at 6 and $12 \mathrm{~h}$ post-scratching, and the experiment was repeated three times.

Tube formation test. A 150- $\mu 1$ 1:1 Matrigel and medium mixture was added into a pre-chilled 24-well plate, and incubated at $37^{\circ} \mathrm{C}$ for $1 \mathrm{~h}$. The cells $\left(2 \times 10^{4}\right)$ treated with control or xanthatin were seeded into the Matrigel-coated 24-well plate and cultured for $6 \mathrm{~h}$. Lumen formation was observed under an inverted microscope. The cells were fixed in 4\% PFA, following which images were captured and the lumen lengths were quantified using ImageJ software version 2X (National Institutes of Health, Bethesda, MD, USA).

Establishment and treatment of the alkali burn model. A total of 60 male adult Sprague-Dawley rats weighing $\sim 180-220 \mathrm{~g}$ were purchased from the Shilaike Experimental Animal Center (Shanghai, China). Following general anesthesia with ether inhalation, all rats were examined for neovascularization, inflammatory response and other lesions. All the rats were administered in the right eye with $1 \%$ tetracaine drops every 2 min for three times for corneal surface anesthesia. A single layer circular filter paper $(3 \mathrm{~mm}$ diameter $)$ was immersed in $1 \mathrm{~mol} / \mathrm{l}$ sodium hydroxide solution for $20 \mathrm{sec}$, and the filter paper was accurately affixed to the central corneal surface of the right eye for $20 \mathrm{sec}$ following the absorbing of excess liquid. The filter paper was then removed from the experimental eye and immediately washed with saline for $1 \mathrm{~min}$. The rats were randomly divided into two groups: A control group and an experimental group, with 30 rats in each group. Following the induction of injury, the control group was treated with PBS (10 $\mu$ l each time, four times per day). In the experimental group, xanthatin solution dissolved in PBS, with a final concentration of $10 \mu \mathrm{M}$, was applied (10 $\mu \mathrm{l}$ each time, four times per day). The course of treatment was 14 days. The corneas were observed at 0,4, 7, 10 and 14 days, and the corneal neovascularization was recorded using a slit lamp to verify the damage of the corneal epithelium. The rats were sacrificed on day 14 of treatment, and the corneas were placed in a freezer $\left(-80^{\circ} \mathrm{C}\right)$ for later experiments. Ethics approval was obtained from the Medical Ethics Committee of The First Affiliated Hospital of Nanchang University (Nanchang, China).

Measurement of corneal neovascularization. The rat corneas were observed with a slit lamp under a microscope at 4, 7, 10 and 14 days, and the area of corneal neovascularization 
was measured based on the vertical length of the longest vessel observed to grow continuously toward the center of the corneal opacity. The formula of corneal neovascularization was calculated according to the Robert model: $\mathrm{S}=\mathrm{C} / 12$ $\mathrm{x} 3.1416 \mathrm{x}\left[\mathrm{r}^{2}-(\mathrm{r}-\mathrm{I})^{2}\right]$, where $\mathrm{C}$ is the time of the circumference of the cornea that accumulates for the new blood vessel (14), $r$ is the corneal radius, and $\mathrm{L}$ is the length of corneal neovascularization from the corneal limbus into the cornea.

Histological examination. The corneas were harvested at the end of the experiment, cut into small pieces and fixed in $4 \%$ PFA. The samples were processed via routine dehydration with ethanol gradient, clearance with xylene, and then embedded in paraffin. The coronal section was cut into $5-\mu \mathrm{m}$ thick tissue sections parched on the slide. The slides were deparaffinized, stained with hematoxylin and eosin, dehydrated with alcohol, and mounted in neutral balsam by using an Eclipse 50i clinical microscope (Nikon Corporation, Tokyo, Japan).

Western blot analysis. The corneal tissues of the rats were removed on day 14 post-alkali burn, ground in $100 \mu 1$ lysis buffer for $20 \mathrm{~min}$, and homogenized on ice three times (30 sec each with intervals of $20 \mathrm{sec}$ ). The homogenate was incubated on ice for $20 \mathrm{~min}$ and centrifuged at $4^{\circ} \mathrm{C}$ at $2,000 \mathrm{x} \mathrm{g}$ for $20 \mathrm{~min}$. Following determination of the protein concentration of the sample using BCA standards, and sampling buffer was added to the protein sample and then boiled for denaturalization. The protein samples of each group were analyzed on a $10 \%$ SDS-PAGE gel and transferred onto a PVDF membrane following electrophoresis. Following blocking in 2\% BSA (Sigma-Aldrich; F Merck KGaA, Darmstadt, Germany) for $1 \mathrm{~h}$ at room temperature, the membrane was incubated with VEGF (cat. no. ab46154; 1:200; Abcam, Cambridge, MA, USA), pigment epithelium-derived factor (PEDF) (cat. no. sc-25594; 1:200; Santa Cruz Biotechnology, Inc., Dallas, TX, USA), phosphorylated (p-)VEGFR2 (cat. no. ab5473; 1:500; Abcam), total (t)VEGFR2 (cat. no. ab10972; 1:500; Abcam), p-signal transducer and activator of transcription 3 (STAT3) (cat. no. ab76315; 1:2,000; Abcam), t-STAT3 (cat. no. ab68153; 1:1,000; Abcam), p-phosphoinositide 3-kinase (PI3K) (cat. no. 4228s; 1:1,000; Cell signaling Technology, Inc., Danvers, MA, USA), t-PI3K (cat. no. ab86714; 1:1,000; Abcam, Cambridge, MA, USA), p-Akt (cat. no. 4060; 1:1,000; Cell signaling Technology, Inc.), t-Akt (cat. no. 4685; 1:1,000; Cell signaling Technology, Inc.) and $\beta$-actin (cat. no. A5316; 1:10,000; Sigma-Aldrich; Merck $\mathrm{KGaA}$ ) antibodies overnight at $4^{\circ} \mathrm{C}$. Once the incubation was complete, the membrane was washed with TBST at room temperature three times on a rocking device (10 min each time) Following incubation for $2 \mathrm{~h}$ with secondary horseradish peroxidase antibody (Goat anti-Rabbit IgG HRP Affinity; cat. no. HAF008; 1:5,000; R\&D Systems, Inc., Minneapolis, MN, USA) at room temperature, the membrane was washed with TBST three times at room temperature $(10 \mathrm{~min}$ each time). Finally, the ECL color solution was added onto the membrane in the darkroom to develop the film. Images were analyzed using ImageJ software to calculate the gray values.

Immunofluorescence detection. Following deparaffinization, the slides were placed in EDTA buffer and boiled in a microwave for antigen retrieval. Following cooling, the slides were washed with PBS three times (5 min each). The slides were then blocked with 5\% BSA for $20 \mathrm{~min}$, and incubated with $50 \mu \mathrm{l}$ diluted primary antibodies against VEGF (cat. no. ab46154; 1:200; Abcam) and PEDF (cat. no. sc-25594; 1:200; Santa Cruz Biotechnology, Inc.) at $4^{\circ} \mathrm{C}$ overnight. Following washing with PBS, $50 \mu 1$ of diluted secondary antibody (goat anti-rabbit IgG; cat. no. F-6005; Lot:065k6224; 1:100, Sigma-Aldrich; Merck $\mathrm{KGaA}$ ) was added to each slide, incubated at room temperature for $1 \mathrm{~h}$ and counterstained with DAPI. The slides were mounted with anti-quenching media and were maintained at $4^{\circ} \mathrm{C}$ in the dark until images were captured using the Leica upright microscope (DM2500; Leica Microsystems $\mathrm{GmbH}$, Wetzlar, Germany).

Statistical analysis. The experimental data were analyzed using SPSS 20.0 (IBM SPSS, Armonk, NY, USA) and all experimental data are expressed as the mean \pm standard deviation. Comparisons between groups were assessed using a post hoc Tukey test and correlation analysis. $\mathrm{P}<0.05$ was considered to indicate a statistically significant difference.

\section{Results}

Effect of xanthatin on the cell proliferation capacity of HUVECs. To investigate the mechanism of xanthatin in corneal neovascularization, the present study investigated the effects of different concentrations of xanthatin on the proliferation of cultured HUVECs. The CCK- 8 experimental results showed no significant inhibition of cell proliferation in the $0.1 \mu \mathrm{M}$ xanthatin group, whereas $0.5,1,2,5,10$ and $20 \mu \mathrm{M}$ xanthatin reduced the HUVEC proliferation rate significantly $(\mathrm{P}<0.05$; Fig. 1A). This indicated that different concentrations of xanthatin significantly affected the proliferation of HUVECs.

The results also showed that the effects of 10 and $20 \mu \mathrm{M}$ xanthatin on the proliferation of HUVECs were equivalent, indicating that $10 \mu \mathrm{M}$ may be the optimal concentration for inhibiting the proliferation of HUVECs.

Effect of xanthatin on the migration of HUVECs. In order to further investigate the association between xanthatin and HUVEC migration ability, a scratch test was performed to detect HUVEC migration in a control group and a $10 \mu \mathrm{M}$ xanthatin treatment group (Fig. 1B). Images of the cells in each group were captured under an optical microscope, and each image was captured in the same region close to the scratch. After 0,6 and $12 \mathrm{~h}$, the gap widths between the two groups were significantly different, suggesting that $10 \mu \mathrm{M}$ xanthatin had a marked effect on inhibiting the HUVEC migration ability (Fig. 1C).

Effect of xanthatin on luminal formation of HUVECs. The in vitro tube forming assay showed that the HUVECs formed several densely arranged luminal structures in each image of the control group, whereas fewer luminal structures were visible in the $10 \mu \mathrm{M}$ xanthatin group, which were arranged more sparsely (Fig. 1D). Statistical analysis was performed on the length of the lumen. The results showed that, compared with the control group, the length of the lumen in the $10 \mu \mathrm{M}$ xanthatin treatment group was $\sim 50 \%$ of the length of lumen in the control group (Fig. 1E). Compared with the control group, 
A

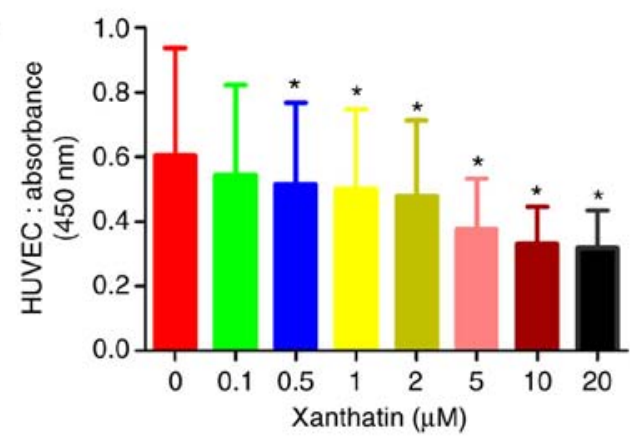

C

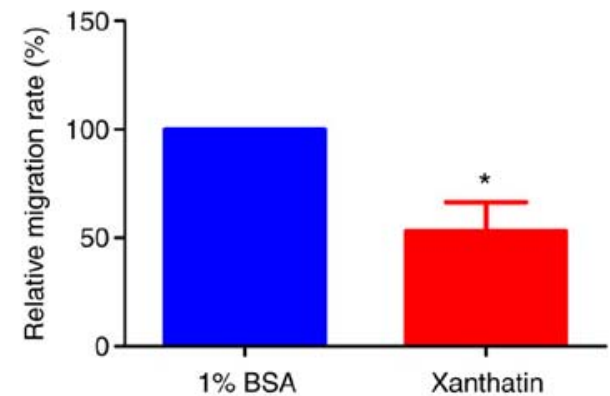

D

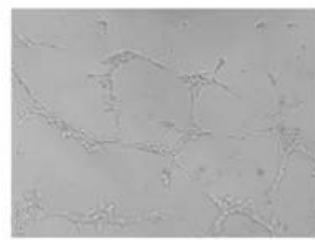

$1 \%$ BSA

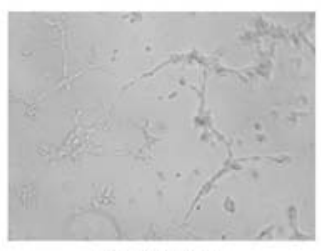

Xanthatin
B
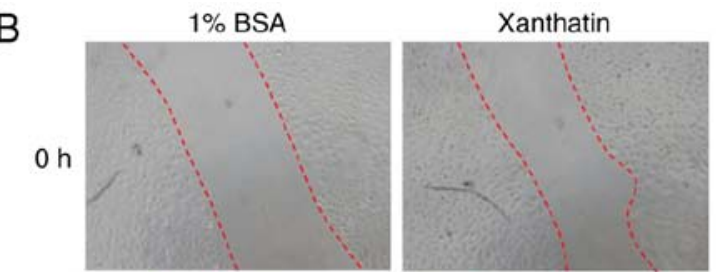

$6 \mathrm{~h}$
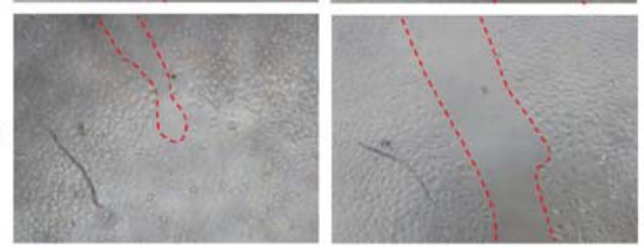

$12 \mathrm{~h}$
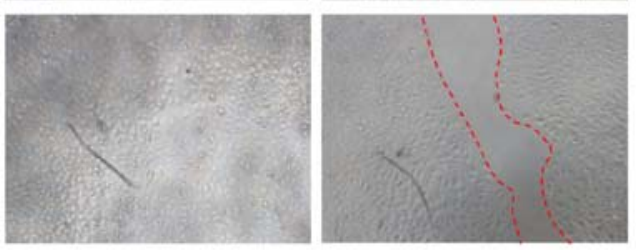

E

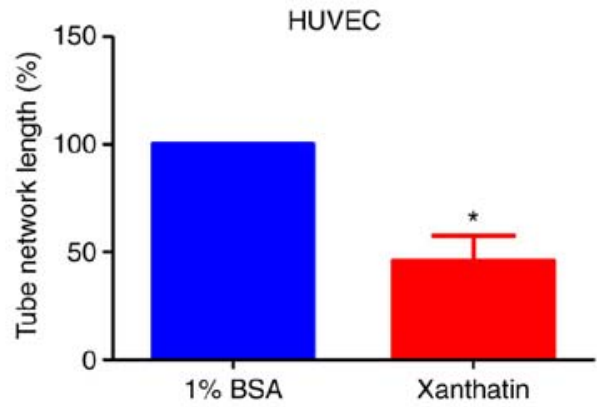

Figure 1. (A) HUVEC proliferation following exposure to different concentrations of xanthatin for $24 \mathrm{~h}$ was detected using a CCK-8 assay. (B) Xanthatin reduced wound closure in a scratch wounding assay of the HUVECs. Images were captured at 0,6 and $12 \mathrm{~h}(\mathrm{magnification}, \mathrm{x} 20)$ and $(\mathrm{C})$ quantified. (D) Effect of xanthatin on tube formation of HUVECs was assessed by a tubulogenesis assay. Images were captured following treatment with xanthatin for $6 \mathrm{~h}$ (E) Representative images from three independent experiments performed in duplicate. All data are presented as the mean \pm standard deviation from three experiments. ${ }^{*} \mathrm{P}<0.05$, compared with the control group. HUVECs, human umbilical vein endothelial cells; BSA, bovine serum albumin.

treatment with $10 \mu \mathrm{M}$ xanthatin significantly inhibited the formation of HUVEC lumen length $(\mathrm{P}<0.05)$.

Analysis of the physiological condition of the alkali burn animal model. Following the establishment of the alkali burn model, the rats were randomly divided into two groups, with 30 rats in each group. One group of rats was administered with PBS eye drops four times a day; the other group was administered with $10 \mu \mathrm{M}$ xanthatin eye drops four times a day (Fig. 2A). The body weights and eye weights of the rates in the two groups were analyzed on days $0,4,7,10$ and 14 (Fig. 2B and C). The results showed no significant differences in body weights between the two groups at different time points, and no significant differences in eye weights were found between the two groups at different time points.

Xanthatin inhibits corneal neovascularization in the alkali burn rat model. In order to examine the effect of xanthatin in corneal neovascularization in an animal model of corneal neovascularization, $10 \mu \mathrm{M}$ xanthatin eye drops were used to treat neovascular keratitis in the corneal alkali burn animal model. Following establishment of the corneal alkali burn model, the rats were administered with $10 \mu \mathrm{M}$ xanthatin eye drops or PBS eye drops for 14 days, following which changes in corneal neovascularization and inflammatory reactions were examined. The corneal slit lamp and corneal tissue changes in the PBS group and the $10 \mu \mathrm{M}$ xanthatin treatment group at 0 and 14 days post-corneal alkali burn are shown in Fig. 3A. In the PBS group, the extent of corneal neovascularization increased significantly with time. By contrast, the corneal neovascularization in the $10 \mu \mathrm{M}$ xanthatin eye drop group regressed in the 14 days. The corneal tissue hematoxylin and eosin staining showed that the number of red blood cells in the corneal stroma layer was increased significantly at 14 days in the PBS group. However, the number of red blood cells in the corneal stromal layer of the $10 \mu \mathrm{M}$ xanthatin treatment group was minimal. The results in the PBS group and the $10 \mu \mathrm{M}$ xanthatin group at $0,4,7$ and 10 days post-alkali burn, and changes in the inflammatory indices in the 14 days are shown in Fig. 3B. The results showed that, in the $10 \mu \mathrm{M}$ xanthatin treatment group, the inflammatory index was significantly lower, compared with that in the PBS eye drop group at each time point. The inflammatory index showed a decreasing trend with time in the $10 \mu \mathrm{M}$ xanthatin treatment group, indicating that $10 \mu \mathrm{M}$ xanthatin effectively controlled the inflammatory response. The area of corneal neovascularization in the PBS 

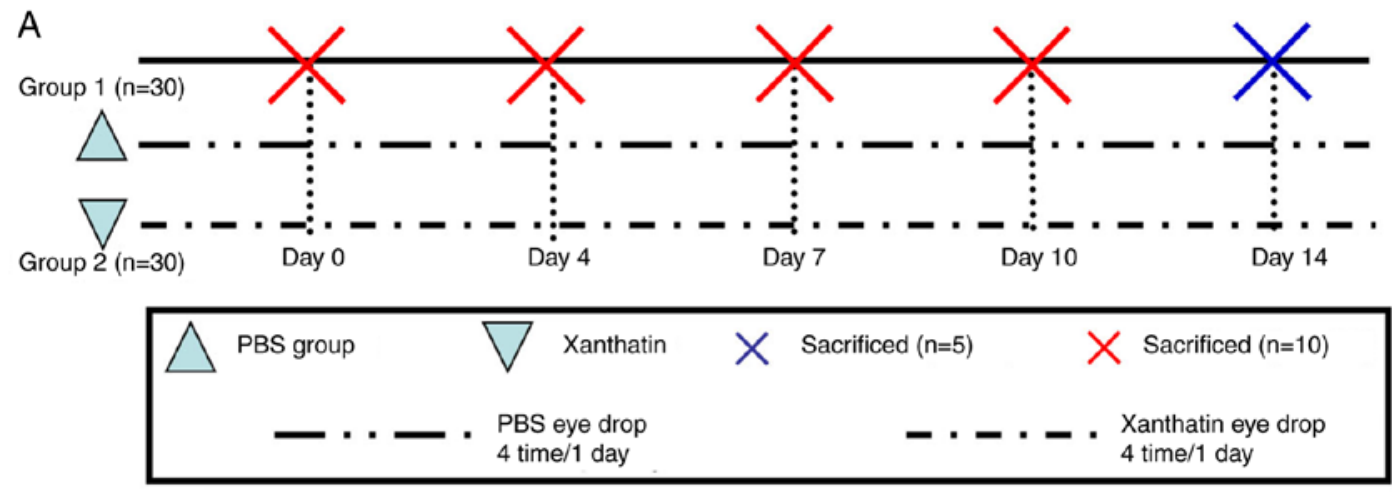

B

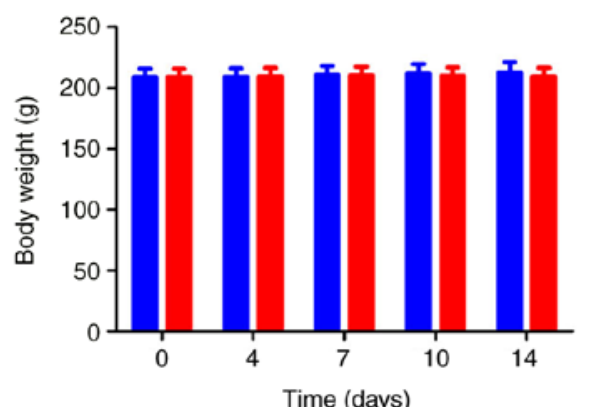

C

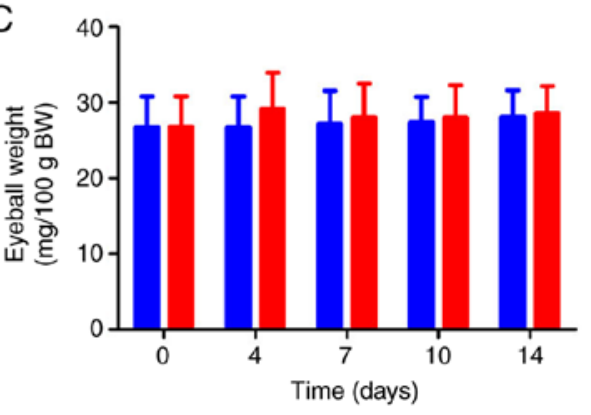

$\square$ PBS $10 \mu \mathrm{M}$ Xanthatin

Figure 2. Experimental design and measurement of parameters. (A) Experiment design of xanthatin treatment on alkali-burned rat corneas. (B) Body weight and (C) eyeball weight of rats in the control group and the xanthatin group were detected at $0,4,7,10$ and 14 days. No differences were found between these two groups. All data are presented as the mean \pm standard deviation from three experiments.

A

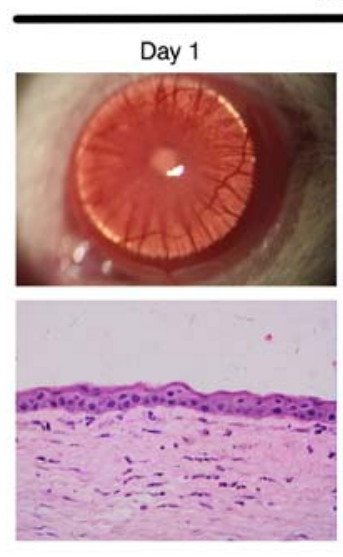

B

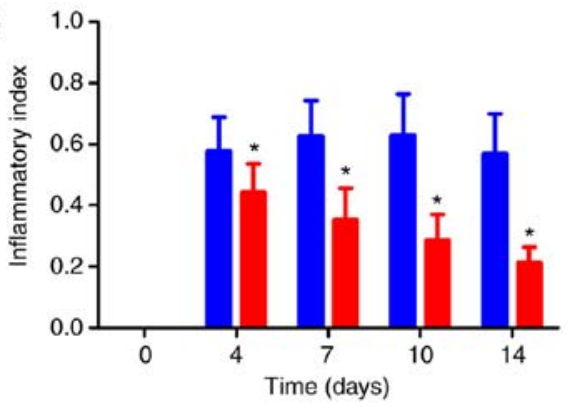

PBS
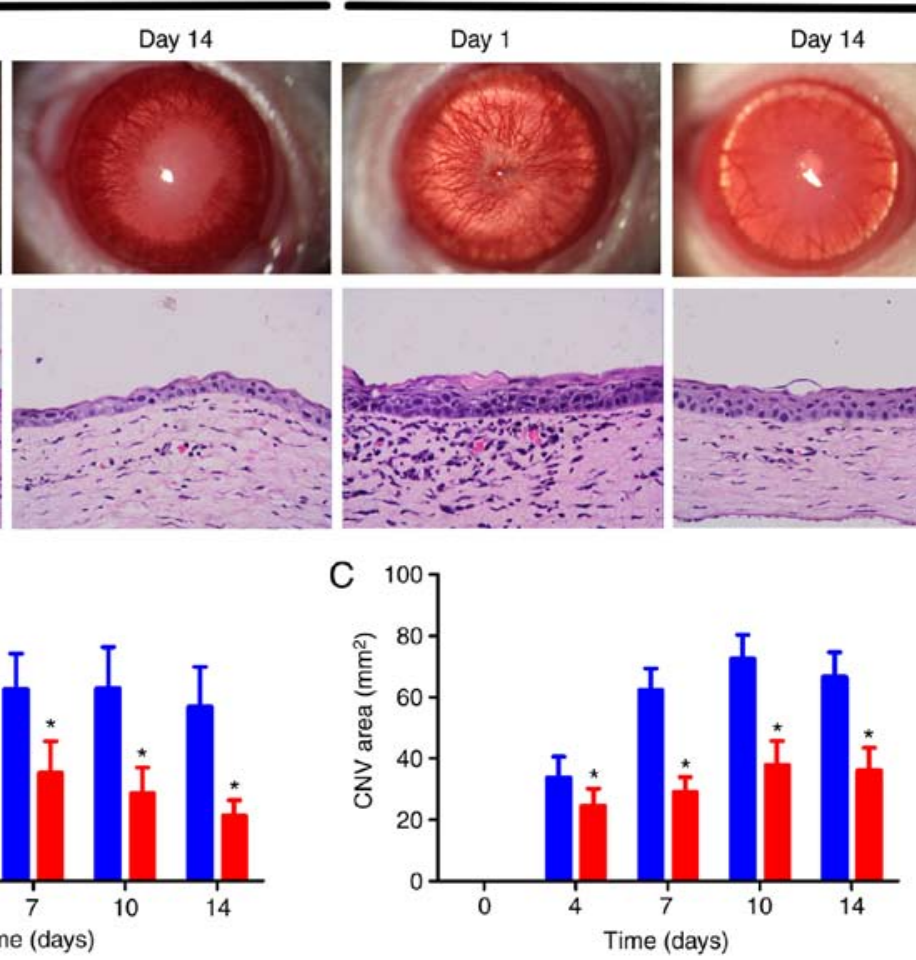

Xanthatin

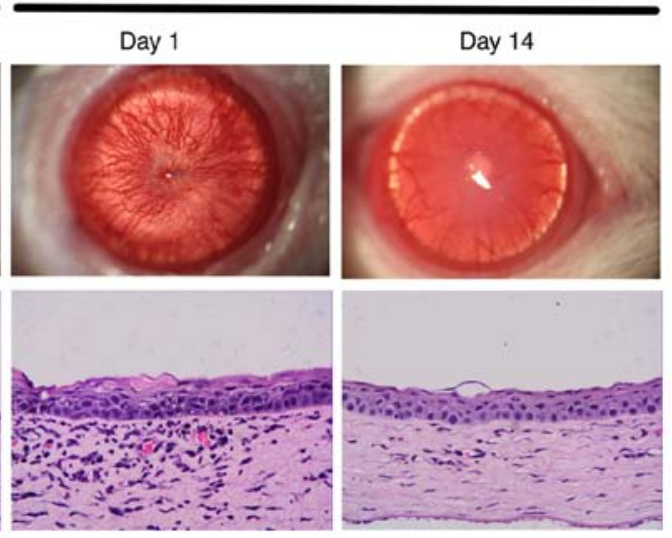

PBS $10 \mu \mathrm{M}$ Xanthatin

Figure 3. Inhibition of alkali burn-induced CNV and inflammation by xanthatin treatment. (A) Stereomicroscopic appearance of mouse eyes of the xanthatin group and the control group on days 1 and 14. Representative hematoxylin and eosin-stained corneal sections of the rats in the xanthatin group and the control group are shown. Magnification, x20. (B) Inflammatory indices of the xanthatin group and the control group were accessed on day 0,4 , 7 , 10 and 14 . (C) A significant regression of the CNV area was observed in the xanthatin group, compared with that in the PBS treatment on days 0,4 , 7,10 and 14 . All data are presented as the mean \pm standard deviation from three experiments. ${ }^{*} \mathrm{P}<0.05$, compared with the control group. $\mathrm{CNV}$, corneal neovascularization; $\mathrm{D}$, day. 

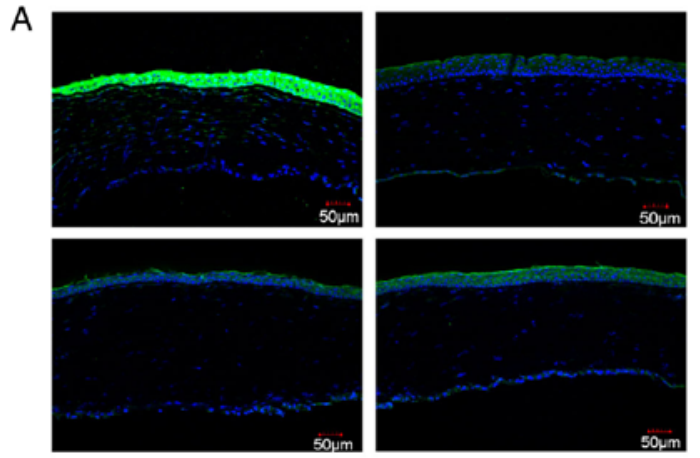

C
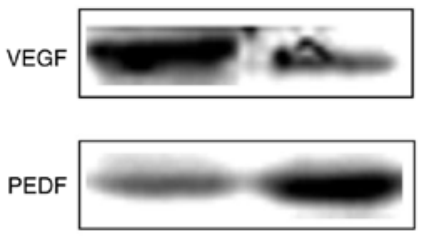

$\beta$-actin

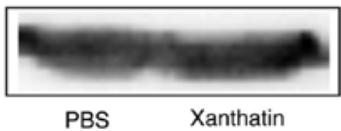

B

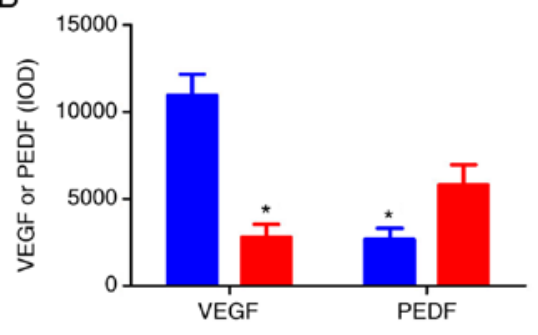

PBS $10 \mu \mathrm{M}$ Xanthatin

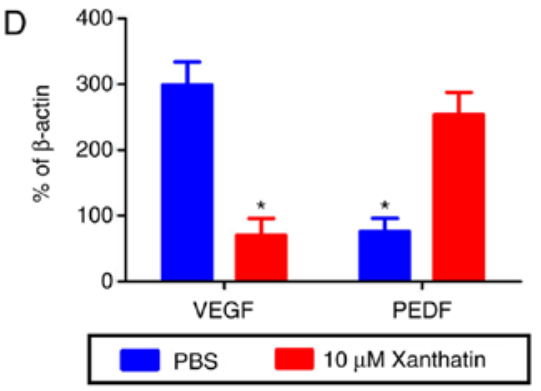

Figure 4. Protein expression of VEGF and PEDF in the xanthatin group and the control group. (A) Representative fluorescence microscopy images showing expression of VEGF and PEDF and their localization in the alkali-burned rat corneas of the xanthatin treatment group and the control group on day 14. (B) Quantification of expression levels of VEGF and PEDF in the xanthatin treatment group and the control group was performed using ImageJ software. (C) Western blot bands of protein expression of VEGF and PEDF in the alkali-burned rat corneas of the xanthatin treatment group and the control group on day 14 are shown. (D) Histograms representing the relative levels of VEGF and PEDF, respectively, in the xanthatin treatment group and the control group. Data are presented as the mean \pm standard deviation from three independent experiments. ${ }^{*} \mathrm{P}<0.05$. VEGF, vascular endothelial growth factor; PEDF, pigment epithelium-derived factor.
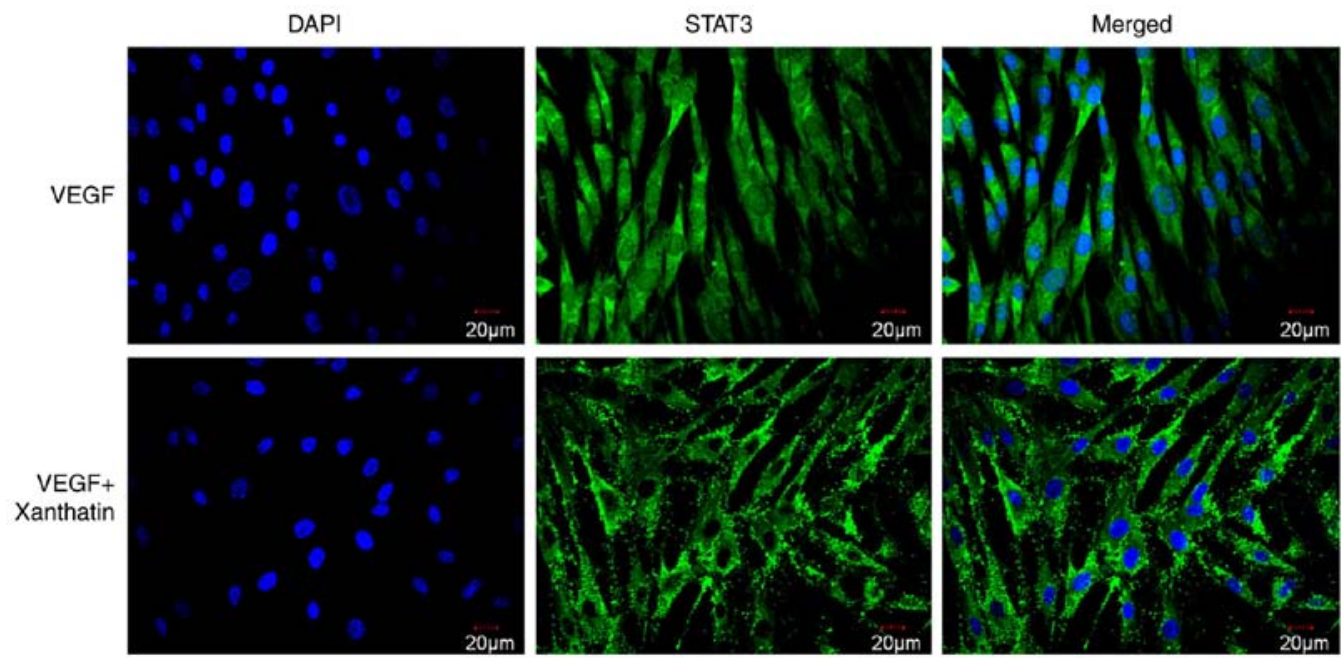

Figure 5. Expression of STAT3 and its subcellular location in VEGF-treated and VEGF+xanthatin-treated human umbilical vein endothelial cells. The nuclei were stained using DAPI. VEGF, vascular endothelial growth factor; STAT3, signal transducer and activator or transcription 3.

group and the $10 \mu \mathrm{M}$ xanthatin group was assessed at 0,4 , 7, 10 and 14 days, respectively (Fig. 3C). The results showed that the area of neovascularization in the $10 \mu \mathrm{M}$ xanthatin treatment group was significantly lower, compared with that in the PBS treatment group, and there were significant differences at each time point. Therefore, $10 \mu \mathrm{M}$ xanthatin inhibited alkali-induced neovascularization.

Xanthatin affects the expression of VEGF and PEDF in the cornea of the alkali burn model. In order to further examine the mechanism of inhibition of corneal neovascularization by xanthatin, western blot analysis and immunofluorescence were used to assess the expression of VEGF and PEDF in the corneal tissue of the PBS group and the $10 \mu \mathrm{M}$ xanthatin treatment group at 14 days post-alkali burn (Fig. 4A-D). The results showed that the expression of VEGF in the cornea increased significantly in the 14 days in the PBS group. However, the expression of VEGF in the corneal tissue was significantly lower in the $10 \mu \mathrm{M}$ xanthatin treatment group, compared with that in the PBS group. The expression level of PEDF in the 
A

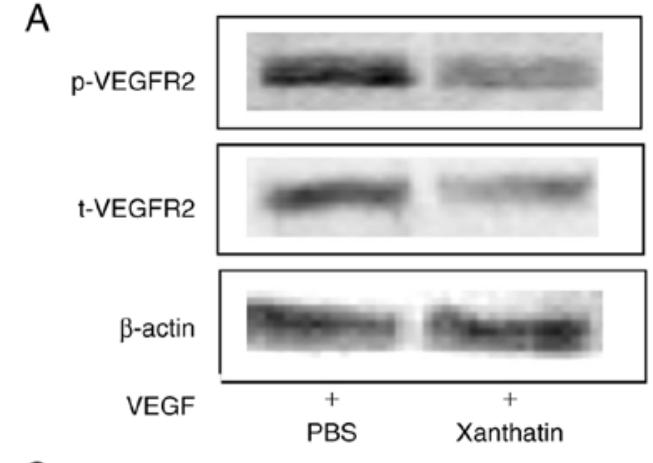

C

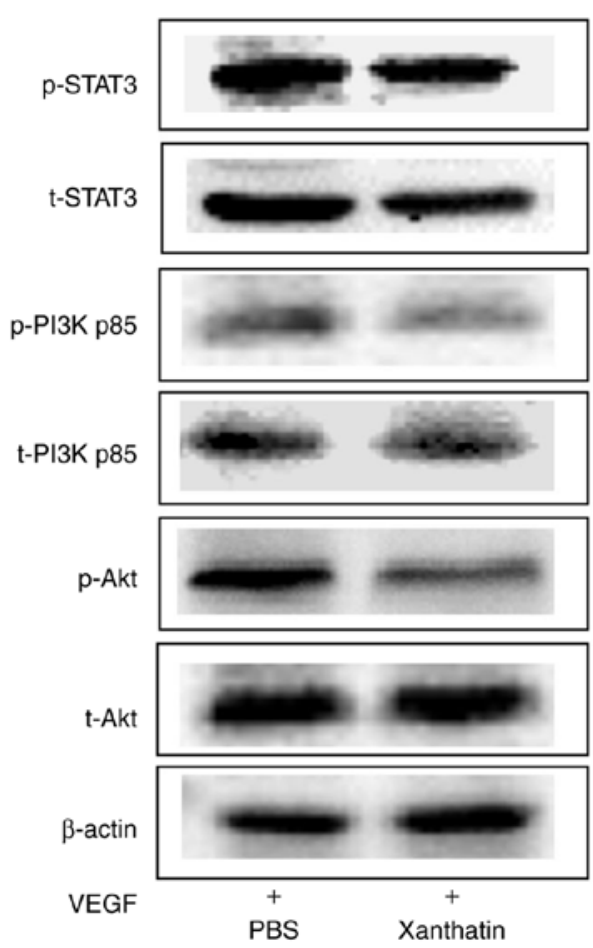

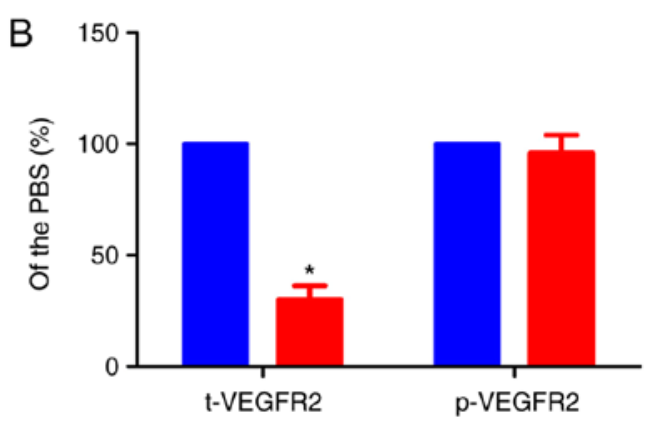

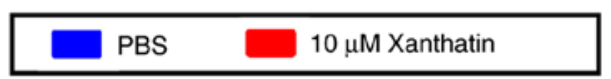

D

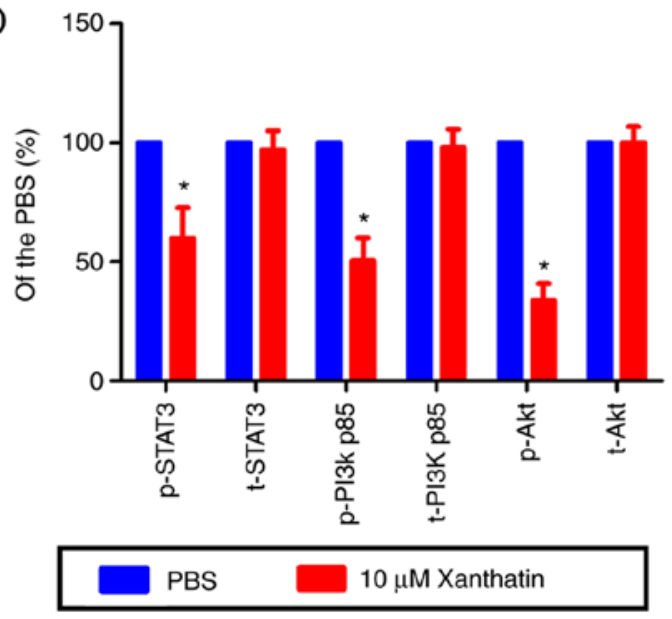

Figure 6. Effect of xanthatin on VEGFR2 and the STAT3/PI3K/Akt pathway in HUVECs. (A) Protein expression levels of p-VEGFR2 and t-VEGFR2 in the control group and the xanthatin group in HUVECs were accessed using western blot analysis. (B) Quantification of western blots for p-VEGFR2 and t-VEGFR2 revealed decreased t-VEGFR2 following treatment of HUEVCs with $10 \mu \mathrm{M}$ xanthatin for $12 \mathrm{~h}$. (C) Expression levels of p-STAT3, t-STAT3, p-PI3K, t-PI3K, p-Akt and t-Akt were detected using western blot analysis and $\beta$-actin was used as a control. (D) Bands of p-STAT3, t-STAT3, p-PI3K, t-PI3K, p-Akt and t-Akt were quantified using ImageJ software and the data are presented as the mean \pm standard deviation from three experiments. $\mathrm{P}<0.05$, compared with the control group. HUVECs, human umbilical vein endothelial cells; VEGFR2, vascular endothelial growth factor receptor; STAT3, signal transducer and activator or transcription 3; PI3K, phosphoinositide 3-kinase; p-, phosphorylated; t-, total.

PBS group decreased significantly in the 14 days, whereas the expression of PEDF in the $10 \mu \mathrm{M}$ xanthatin group was significantly higher, compared with that in the PBS group. Therefore, the inhibition of corneal neovascularization by xanthatin in the alkali burn model was achieved by the downregulation of VEGF and upregulation of PEDF.

Xanthatin inhibits corneal neovascularization through VEGFR2/STAT3/PI3K. To elucidate the mechanisms underlying the inhibition of corneal neovascularization by xanthatin, the present study examined the expression of VEGFR2, an important receptor of VEGF, and its downstream associated molecules. The subcellular localization of STAT3 was detected in HUVECs using immunofluorescence under different conditions. A higher protein expression level of STAT3 was found in the VEGF-treated HUVECs, compared with that in the xanthatin+VEGF-treated HUVECs, in which the protein expression level of STAT3 decreased significantly (Fig. 5). It was concluded that xanthatin significantly downregulated the nuclear expression level of STAT3 in the VEGF-treated HUVECs. The expression levels of VEGFR2 were also compared between the two experimental groups using western blot analysis (Fig. 6A and B). The results showed that the expression level of t-VEGFR2 in the xanthatin-treated group was significantly lower, compared with that in the PBS group, whereas no significant difference was observed in the protein expression level of p-VEGFR2. The expression levels of signaling molecules downstream of VEGFR2, including STAT3, PI3K and Akt, were also examined (Fig. 6C and D). The results showed that the expression levels of p-STAT3, $\mathrm{p}-\mathrm{PI} 3 \mathrm{~K}$ and $\mathrm{p}-\mathrm{Akt}$ in the xanthatin group were significantly lower, compared with those in the PBS group. However, no significant differences were observed in the expression levels of t-STAT3, t-PI3K or t-Akt between the two experimental 


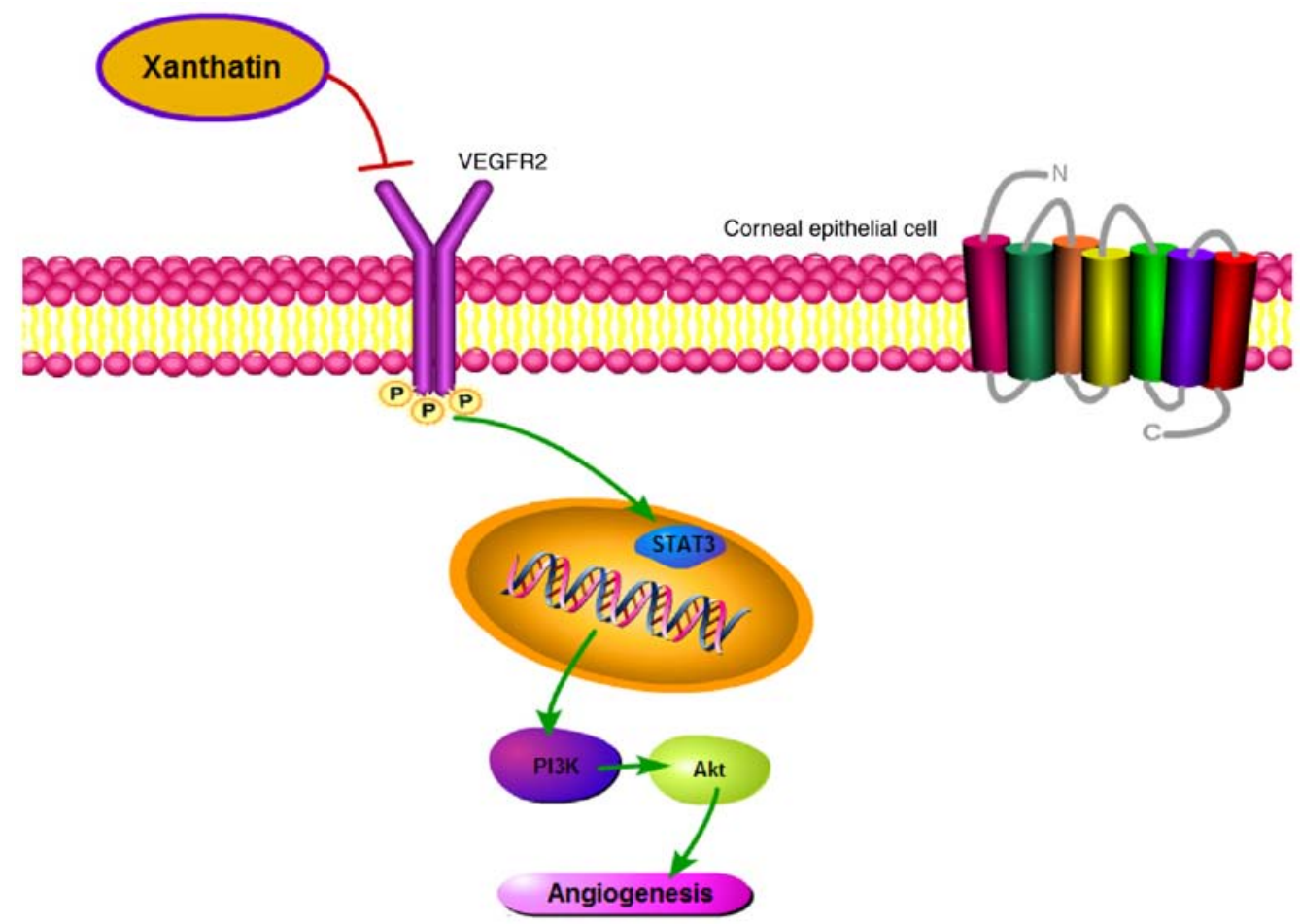

Figure 7. Proposed model illustrating the role of xanthatin and its mechanism in angiogenesis. Xanthatin inhibits corneal neovascularization via downregulation of VEGFR2 and the STAT3/PI3K/Akt signaling pathway. Yellow circles labeled 'P' represent phosphate-VEGFR2. VEGFR2, vascular endothelial growth factor receptor; STAT3, signal transducer and activator or transcription 3; PI3K, phosphoinositide 3-kinase.

groups. It was concluded that xanthatin inhibited corneal neovascularization following alkali burn through the VEGFR2/STAT3/PI3K signaling pathway (Fig. 7).

\section{Discussion}

Normal corneal tissue is completely transparent and has no blood vessels. The surrounding vessels terminate at the limbus and form a vascular network, which provides nutrition to the cornea. The absence of vascularization is the main feature of the cornea. Infection, trauma, transplantation rejection and other factors can disrupt the balance of corneal avascularity and induce the process of angiogenesis (15). The changes of the cornea external environment can cause the formation of new blood vessels (16). Corneal neovascularization often leads to loss of corneal transparency (17). It is an important cause of blindness, and it is also a high-risk factor in rejection following corneal transplantation (18). The effective control of corneal inflammation and neovascularization in corneal diseases has become the main direction of treatment.

Xanthatin is the effective bioactive constituent of Xanthium sibiricum. In Chinese medicine, it is reported to be important in the treatment of colds, limb spasms and ulcer sores, and has antitumor, antiviral and antifungal effects (4). Compared with conventional anticancer therapy, the first generation of anti-angiogeneic drugs currently in clinical development have no clinical effects, therefore, the development of a more potent angiogenic inhibitor has become a promising focus of research. Studies have shown that xanthatin can inhibit tumor angiogenesis, limit its nutritional supply, inhibit tumor cell proliferation and induce the apoptosis of tumor cells, therefore, it can be used as a novel type of antitumor drug (19).
Xanthatin (xanthium pavilion) has been shown to exhibit high in vitro cytotoxic activity in mouse lymphocytic leukemia cell lines (P-388 and L-1210) and human bronchial epidermoid tumor cells (NSCL-N6). However, in in vivo experiments, it exhibited weak cytotoxic activity (20). Previous experimental results show that xanthatin can inhibit the proliferation of HUVECs (12). The present study examined the effects of xanthatin on the migration properties and luminal formation of HUVECs. The results showed that xanthatin inhibited the migration of HUVECs, the number of luminal formations and the length of HUVECs. These results support the hypothesis that xanthatin can inhibit corneal neovascularization. In order to confirm these findings, based on the classic rat corneal alkali burn model, the present study used xanthatin eye drops as the route of treatment to confirm the effects and mechanism underlying the effects of xanthatin on the inhibition of inflammation and angiogenesis. The results showed that the extent of corneal neovascularization in the xanthatin treatment group was decreased significantly in the alkali-burned rats, compared with that in the control group. Using hematoxylin and eosin staining for the analysis of corneal tissues, the results showed that, in 14 days, the corneal stroma of the PBS eye drop-treated rats exhibited numerous red blood cells, whereas no obvious red blood cells were visible in the corneal stroma of the xanthatin treatment group. The inflammatory index was also used as a monitoring index. The results showed that the inflammatory index of the cornea decreased gradually with the prolongation of time, whereas no significant decrease was observed in the inflammatory index of the PBS eye group. The inflammatory index of the xanthatin eye drop group was significantly different from that of the PBS eye drop group at each time point. The area of corneal neovascularization area 
in the xanthatin-treated group was significantly smaller than that of the PBS group, and there were significant differences at each time point. Therefore, xanthatin inhibited neovascularization and inflammation in the corneas of the alkali-burned rats.

Previous studies have confirmed that VEGF and PEDF in the normal cornea are positively expressed, but that the normal cornea maintains transparency and avascularity, possibly due to the anti-angiogenic factor PEDF and the effect of angiogenesis induced by VEGF $(15,21)$. Therefore, VEGF and PEDG are important in the formation of neovascularization following alkali burn. Under normal physiological conditions, there is a dynamic balance between VEGF and PEDF, which prevents neovascularization (22). In the present study, the expression levels of VEGF and PEDF in the corneal tissues of rats exposed to alkali burn were detected using immunofluorescence and western blot analysis. The results showed that, compared with the PBS eye drop group, the expression level of VEGF in the cornea of the xanthatin eye drop group was markedly decreased, whereas the expression level of PEDF was significantly increased. These results suggested that xanthatin inhibited neovascularization in the cornea of alkali-burned rats via the downregulation of VEGF and upregulation of PEDF.

Angiogenesis occurs through endothelial cell proliferation, differentiation, migration and lumen formation. This process is caused by a variety of factors, and VEGF is one of the most important regulatory factors. The VEGFR family is composed of three receptors, VEGFR1, VEGFR2 and VEGFR3, and VEGFR2 is involved in the main process of mediating VEGF-induced neovascularization (23). It has been shown that the VEGF/VEGFR2 signaling pathway is key in corneal neovascularization (24). In the present study, xanthatin significantly inhibited the expression of VEGF in the cornea of alkali-burn model rats. In the in vitro experiments, it was confirmed that xanthatin also downregulated the VEGF-induced expression level of VEGFR2 in HUVECs. From this, it was concluded that xanthatin regulated corneal neovascularization by regulating the VEGF/VEGFR2 signaling pathway.

STAT3 is a transcription factor, which can be activated by a variety of cytokines and growth factors. Following activation, STAT3 is translocated into the nucleus and regulates apoptosis, proliferation, migration and the expression of associated genes in the nucleus (25). Studies have shown that inhibition of the STAT3 signaling pathway mediated by VEGFR2 is an effective way of inhibiting neovascularization and tumor proliferation $(26,27)$. In tumor vascular endothelial cells, the VEGFR2 pathway upregulates STAT3, causing the translocation of STAT3 into the nucleus, resulting in tumor angiogenesis (28). In retinal microvascular endothelial cells, small interfering RNA-mediated knockdown inhibits the activation of VEGFR2 and phosphorylation of STAT3 protein, which inhibits the proliferation of microvascular endothelial cells and the formation of pathological neovascularization (29). In a murine melanoma model, inhibition of the protein expression of VEGFR2 downregulated the phosphorylation of STAT3 protein and its nuclear localization, which inhibited tumor proliferation and neovascularization (30). Therefore, the VEGFR2/STAT3 signaling pathway is important in angiogenesis. In the present study, the effects of xanthatin on the subcellular localization and the phosphorylation level of STAT3 were determined in VEGF-induced HUVECs. The results showed that xanthatin significantly reduced the intracellular localization of STAT3 in the HUVECs and a significant decrease in the phosphorylation level of STAT3 was detected. It was concluded that xanthatin regulated the VEGFR2/STAT3 signaling pathway in the VEGF-induced HUVECs, and reduced the level and phosphorylation of nuclear STAT3, reducing the occurrence of neovascularization.

The PI3K/Akt signaling pathway is involved in a variety of important cellular processes, including cell growth, metabolism, survival and angiogenesis (31). This pathway is also key in the regulation of neovascularization by $\operatorname{VEGF}(32,33)$. The PI3K/Akt signaling pathway inhibits angiogenesis in malignant liver tumors, melanoma, hemangioma and renal cell carcinoma by regulating vascular endothelial remodeling (34-37). The PI3K/Akt signaling pathway is an important downstream pathway of STAT3 protein, and the present study hypothesized that xanthatin inhibits corneal neovascularization by inhibiting the PI3K/Akt signaling pathway regulated by STAT3. Therefore, the present study investigated whether xanthatin affected the expression of the PI3K/Akt signaling pathway in VEGF-induced HUVECs. The results showed that xanthatin significantly inhibited the phosphorylation levels of PI3K and Akt. It was concluded that xanthatin inhibited the luminal formation and proliferation of HUVECs induced by VEGF through inhibition of the PI3K/Akt signaling pathway. Corneal neovascularization was inhibited by xanthatin in the alkali burn model, possibly through the same mechanism.

In conclusion, xanthatin effectively inhibited the formation of corneal neovascularization in alkali burn rats. The primary mechanism underlying the effect of xanthatin may be through downregulating the expression level of VEGFR2 to inhibit the nuclear localization and protein phosphorylation of STAT3, and inhibit the downstream activation of the PI3K/Akt signaling pathway. Xanthatin is expected to become a novel drug for inhibiting neovascularization in ophthalmology and other associated disciplines, providing novel direction in studies investigating the inhibition of neovascularization.

\section{Acknowledgements}

Not applicable.

\section{Funding}

This study was supported by grants from the National Natural Science Foundation of China (grant nos. 81160118, 81460092, 81460372 and 81660152) to Professor Yi Shao.

\section{Availability of data and material}

The datasets used and/or analyzed during the current study are available from the corresponding author on reasonable request.

\section{Authors' contributions}

MS and YS conceived the concept of the study. LY and XZZ designed the study. QY, XZZ, QCY and CS analyzed the experiment data. MS, PWZ, NJ and MYM performed the experiment. $\mathrm{XZZ}$ wrote the manuscript and was involved in 
revising it critically for important intellectual content. All authors discussed the results and implications and commented on the manuscript at all stages.

\section{Ethics approval and consent to participate}

The present study was approved by the Medical Ethics Committee of The First Affiliated Hospital of Nanchang University (Nanchang, China).

\section{Consent for publication}

Not applicable.

\section{Competing interests}

The authors declare that they have no competing interests.

\section{References}

1. Ellenberg D, Azar DT, Hallak JA, Tobaigy F, Han KY, Jain S, Zhou Z and Chang JH: Novel aspects of corneal angiogenic and lymphangiogenic privilege. Prog Retin Eye Res 29: 208-248, 2010.

2. West SK: Blinding trachoma: Prevention with the safe strategy. Am J Trop Med Hyg 69 (5 Suppl): S18-S23, 2003

3. Tutin TG, Heywood VH, Burges NA, Moore DM, Valentine DH, Walters SM and Webb DA: Flora Europea. Volume 4. Plantaginaceae to Compositae (and Rubiaceae). Cambridge University Press, Cambridge, 1976.

4. Romero M, Zanuy M, Rosell E, Cascante M, Piulats J, Font-Bardia M, Balzarini J, De Clerq E and Pujol MD: Optimization of xanthatin extraction from Xanthium spinosum L. and its cytotoxic, anti-angiogenesis and antiviral properties. Eur J Med Chem 90: 491-496, 2015.

5. Li WD, Wu Y, Zhang L, Yan LG, Yin FZ, Ruan JS, Chen ZP, Yang GM, Yan CP, Zhao D, et al: Characterization of xanthatin: Anticancer properties and mechanisms of inhibited murine melanoma in vitro and in vivo. Phytomedicine 20: 865-873, 2013.

6. Tao L, Sheng X, Zhang L, Li W, Wei Z, Zhu P, Zhang F, Wang A, Woodgett JR and Lu Y: Xanthatin anti-tumor cytotoxicity is mediated via glycogen synthase kinase- $3 \beta$ and $\beta$-catenin. Biochem Pharmacol 115: 18-27, 2016.

7. Pinel B, Landreau A, Seraphin D, Larcher G, Bouchara JP and Richomme P: Synthesis of reduced xanthatin derivatives and in vitro evaluation of their antifungal activity. J Enzyme Inhib Med Chem 20: 575-579, 2005.

8. Chandel S, Bagai U and Vashishat N: Antiplasmodial activity of Xanthium strumarium against Plasmodium berghei-infected BALB/c mice. Parasitol Res 110: 1179-1183, 2012.

9. Waltenberger J, Claesson-Welsh L, Siegbahn A, Shibuya M and Heldin CH: Different signal transduction properties of KDR and Flt1, two receptors for vascular endothelial growth factor. J Bio Chem 269: 26988-26995, 1994.

10. Quinn TP, Peters KG, De Vries C, Ferrara N and Williams LT: Fetal liver kinase 1 is a receptor for vascular endothelial growth factor and is selectively expressed in vascular endothelium. Proc Natl Acad Sci USA 90: 7533-7537, 1993.

11. Gille H, Kowalski J, Li B, LeCouter J, Moffat B, Zioncheck TF, Pelletier N and Ferrara N: Analysis of biological effects and signaling properties of Flt-1 (VEGFR-1) and KDR (VEGFR-2). $A$ reassessment using novel receptor-specific vascular endothelial growth factor mutants. J Biol Chem 276: 3222-3230, 2001.

12. Bernatchez PN, Soker S and Sirois MG: Vascular endothelial growth factor effect on endothelial cell proliferation, migration, and platelet-activating factor synthesis is Flk-1-dependent. J Biol Chem 274: 31047-31054, 1999.

13. Yu Y, Yu J, Pei CG, Li YY, Tu P, Gao GP and Shao Y: Xanthatin, a novel potent inhibitor of VEGFR2 signaling, inhibits angiogenesis and tumor growth in breast cancer cells. Int J Clin Exp Pathol 8: 10355-10364, 2015.
14. D'Amato RJ, Loughnan M, Flynn E and Folkman J: Thalidomide is an inhibitor of angiogenesis. Proc Natl Acad Sci USA 91: 4082-4085, 1994.

15. Chang JH, Garg NK, Lunde E, Han KY, Jain S and Azar DT: Corneal neovascularization: An anti-VEGF therapy review. Surv Ophthalmol 57: 415-429, 2012.

16. Eliason JA: Leukocytes and experimental corneal vascularization. Invest Ophthalmol Vis Sci 17: 1087-1095, 1978.

17. Qazi Y, Wong G, Monson B, Stringham J and Ambati BK: Corneal transparency: Genesis, maintenance and dysfunction. Brain Res Bull 81: 198-210, 2010.

18. Coster DJ and Williams KA: The impact of corneal allograft rejection on the long-term outcome of corneal transplantation. Am J Ophthalmol 140: 1112-1122, 2005.

19. Nibret E, Youns M, Krauth-Siegel RL and Wink M: Biological activities of xanthatin from xanthium strumarium leaves. Phytother Res 25: 1883-1890, 2011.

20. Wang L, Wang J, Li F, Liu X, Chen B, Tang YX and Wang MK: Cytotoxic sesquiterpene lactones from aerial parts of xanthium sibiricum. Planta Med 79: 661-665, 2013.

21. Matsui T, Nishino Y, Maeda S and Yamagishi S: PEDF-derived peptide inhibits corneal angiogenesis by suppressing VEGF expression. Microvasc Rec 84: 105-108, 2012.

22. Roussakis C, Chinou I, Vayas C, Harvala C and Verbist JF: Cytotoxic activity of xanthatin and the crude extracts of xanthium strumarium. Planta Med 60: 473-474, 1994.

23. Ohno-Matsui K, Morita I, Tombran-Tink J, Mrazek D, Onodera M, Uetama T, Hayano M, Murota SI and Mochizuki M: Novel mechanism for age-related macular degeneration: An equilibrium shift between the angiogenesis factors VEGF and PEDF. J Cell Physiol 189: 323-333, 2001.

24. Ferrara N: Vascular endothelial growth factor: Basic science and clinical progress. Endocr Rev 25: 581-611, 2004.

25. Chen WL, Chen YM, Chu HS, Lin CT, Chow LP, Chen CT and Hu FR: Mechanisms controlling the effects of bevacizumab (avastin) on the inhibition of early but not late formed corneal neovascularization. PLoS One 9: e94205, 2014.

26. Kortylewski M and $\mathrm{Yu} \mathrm{H}$ : Role of Stat 3 in suppressing anti-tumor immunity. Curr Opin Immunol 20: 228-233, 2008.

27. Vesely DL: Cardiac hormones for the treatment of cancer. Endocr Relat Cancer 20: R113-R125, 2013.

28. Vesely DL: Family of peptides synthesized in the human body have anticancer effects. Anticancer Res 34: 1459-1466, 2014.

29. Arulanandam R, Batenchuk C, Angarita FA, Ottolino-Perry K, Cousineau S, Mottashed A, Burgess E, Falls TJ, De Silva N, Tsang J, et al: VEGF-mediated induction of PRD1-BF1/Blimp1 expression sensitizes tumor vasculature to oncolytic virus infection. Cancer Cell 28: 210-224, 2015.

30. Yang Z, Wang H, Jiang Y and Hartnett ME: VEGFA activates erythropoietin receptor and enhances VEGFR2-mediated pathological angiogenesis. Am J Pathol 184: 1230-1239, 2014.

31. Kamran MZ and Gude RP: Pentoxifylline inhibits melanoma tumor growth and angiogenesis by targeting STAT3 signaling pathway. Biomed Pharmacother 67: 399-405, 2013.

32. Marone R, Cmiljanovic V, Giese B and Wymann MP: Targeting phosphoinositide 3-kinase: Moving towards therapy. Biochim Biophys Acta 1784: 159-185, 2008.

33. Cai J, Ahmad S, Jiang WG, Huang J, Kontos CD, Boulton M and Ahmed A: Activation of vascular endothelial growth factor receptor- 1 sustains angiogenesis and $\mathrm{Bcl}-2$ expression via the phosphatidylinositol 3-kinase pathway in endothelial cells. Diabetes 52: 2959-2968, 2003.

34. Alvarez Y, Astudillo O, Jensen L, Reynolds AL, Waghorne N, Brazil DP, Cao Y, O'Connor JJ and Kennedy BN: Selective inhibition of retinal angiogenesis by targeting PI3 kinase. PLoS One 4: e7867, 2009.

35. Ji Y, Chen S, Li K, Li L, Xu C and Xiang B: Signaling pathways in the development of infantile hemangioma. J Hematol Oncol 7: 13, 2014.

36. Posadas EM, Limvorasak $S$, Sharma $S$ and Figlin RA: Targeting angiogenesis in renal cell carcinoma. Expert Opin Pharmacother 14: 2221-2236, 2013.

37. Zhu YJ, Zheng B, Wang HY and Chen L: New knowledge of the mechanisms of sorafenib resistance in liver cancer. Acta Pharmacol Sin 38: 614-622, 2017.

This work is licensed under a Creative Commons Attribution-NonCommercial-NoDerivatives 4.0 International (CC BY-NC-ND 4.0) License. 